\title{
The effect of surgical case volume on outcome after the Norwood procedure
}

\author{
Paul A. Checchia, MD, FAAP ${ }^{a, b}$ \\ Jamie McCollegan, BS ${ }^{\mathrm{C}}$ \\ Noha Daher, MS ${ }^{\mathrm{d}}$ \\ Nikoleta Kolovos, MDa \\ Fiona Levy, MD, MBA ${ }^{\text {a }}$ \\ Barry Markovitz, MD, MPH ${ }^{\mathrm{a}, \mathrm{c}}$
}

From the Divisions of Critical Care Medicine $^{\mathrm{a}}$ and Cardiology $\mathrm{y}^{\mathrm{b}}$ and the Departments of Pediatrics and Anesthesiology, ${ }^{\mathrm{c}}$ Washington University School of Medicine, St Louis, Mo, Children's Hospital, St Louis, Mo, and the School of Allied Health Professionals, ${ }^{\mathrm{d}}$ Loma Linda University, Loma Linda, Calif.

Received for publication April 27, 2004; revisions received June 24, 2004; accepted for publication July 8, 2004.

Address for reprints: Paul A. Checchia, MD, FAAP, Washington University School of Medicine, St Louis Children's Hospital, Campus Box 8116, One Children's Place, Suite 5S20, St Louis, MO 63110. (E-mail: pchecchia@wustl.edu)

J Thorac Cardiovasc Surg 2005;129:754-9 $0022-5223 / \$ 30.00$

Copyright (C) 2005 by The American Association for Thoracic Surgery

doi:10.1016/j.jtcvs.2004.07.056
Objectives: We analyze the effect of surgical case volume on the survival of infants with hypoplastic left heart syndrome who underwent stage I surgical palliation (the Norwood procedure). The purpose of our study was to understand more clearly the relative effects of institution and surgeon experience on patient outcome.

Methods: Using the Pediatric Health Information System database belonging to the pediatric hospital members of the Child Health Corporation of America, we identified newborn infants $(<30$ days old on admission) from 1998 through 2001 admitted with the diagnosis of hypoplastic left heart syndrome. Stepwise multiple regression analysis was used to examine the association between both institutional and surgeon case volume with 28-day survival after the Norwood procedure.

Results: Twenty-nine hospitals and 87 surgeons performed 801 Norwood procedures during the study period. In the 4 of 29 institutions that averaged 1 or more Norwood procedures per month during the study period, survival averaged $78 \%$. The remaining 25 institutions averaged 1 Norwood procedure every 9.6 weeks, with a survival of $59 \%$. Data analysis revealed that higher institutional volume $(P=.02)$ but not the number of cases performed by surgeons $(P=.13)$ increased survival after the Norwood procedure. There was no such association with average length of stay in survivors or the time to mortality in nonsurvivors.

Conclusion: Survival after the Norwood procedure is associated with institutional Norwood procedure volume but not with individual surgeon case volume, suggesting the need for improvements in institutional-based approaches to the care of children with hypoplastic left heart syndrome and the need for establishing regional referral centers for such high-risk procedures to improve patient survival.

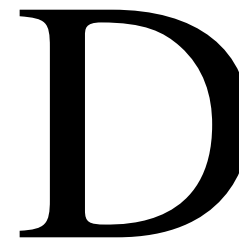

espite dramatic improvements in both cardiac transplantation and staged reconstructive approach, the perioperative mortality and morbidity associated with the stage I palliation (Norwood procedure) of hypoplastic left heart syndrome (HLHS) remains high. ${ }^{1}$ Although attempts have been made to improve the outcome of stage I reconstruction through improved patient selection and postoperative care on the basis of anatomic and physiologic factors, ${ }^{2-9}$ no one set of characteristics has been consistently predictive of better outcomes, and all such reports have been uncontrolled case series from which contributing factors cannot be discerned. Rather, these improved outcomes have been largely reflective of the commitment and efforts of individual centers and their surgeons, anesthesiologists, intensivists, nurses, and other key personnel. 
TABLE 1. Institutional characteristics

\begin{tabular}{|c|c|c|c|}
\hline Institution & $\begin{array}{c}\text { No. of } \\
\text { Norwood } \\
\text { procedures } \\
\text { performed }\end{array}$ & $\begin{array}{c}\text { Proportion of } \\
\text { Norwood } \\
\text { survival }\end{array}$ & $\begin{array}{c}\text { No. of } \\
\text { surgeons } \\
\text { performing } \\
\text { the } \\
\text { Norwood } \\
\text { procedure }\end{array}$ \\
\hline \multicolumn{4}{|c|}{$\begin{array}{l}\text { Low volume }(<16 \\
\text { cases) }\end{array}$} \\
\hline 1 & 2 & $50 \%$ & 1 \\
\hline 2 & 4 & $25 \%$ & 2 \\
\hline 3 & 4 & $25 \%$ & 1 \\
\hline 4 & 7 & $57 \%$ & 1 \\
\hline 5 & 11 & $36 \%$ & 2 \\
\hline 6 & 11 & $100 \%$ & 5 \\
\hline 7 & 12 & $83 \%$ & 2 \\
\hline 8 & 13 & $38 \%$ & 3 \\
\hline 9 & 15 & $40 \%$ & 2 \\
\hline Average & 8.8 & $48 \%$ & 2.1 \\
\hline \multicolumn{4}{|c|}{$\begin{array}{l}\text { Medium volume (16- } \\
30 \text { cases) }\end{array}$} \\
\hline 10 & 16 & $31 \%$ & 1 \\
\hline 11 & 16 & $56 \%$ & 1 \\
\hline 12 & 18 & $82 \%$ & 4 \\
\hline 13 & 20 & $70 \%$ & 2 \\
\hline 14 & 20 & $90 \%$ & 1 \\
\hline 15 & 21 & $43 \%$ & 2 \\
\hline 16 & 21 & $76 \%$ & 2 \\
\hline 17 & 24 & $37 \%$ & 5 \\
\hline 18 & 25 & $68 \%$ & 2 \\
\hline 19 & 26 & $65 \%$ & 4 \\
\hline Average & 20.7 & $62 \%$ & 2.4 \\
\hline \multicolumn{4}{|c|}{$\begin{array}{l}\text { Higher volume (>30 } \\
\text { cases) }\end{array}$} \\
\hline 20 & 31 & $61 \%$ & 2 \\
\hline 21 & 31 & $55 \%$ & 5 \\
\hline 22 & 34 & $68 \%$ & 4 \\
\hline 23 & 38 & $71 \%$ & 3 \\
\hline 24 & 38 & $71 \%$ & 2 \\
\hline 25 & 42 & $69 \%$ & 6 \\
\hline 26 & 53 & $77 \%$ & 2 \\
\hline 27 & 63 & $92 \%$ & 3 \\
\hline 28 & 76 & $72 \%$ & 6 \\
\hline 29 & 109 & $73 \%$ & 5 \\
\hline Average & 51.5 & $71 \%$ & 3.8 \\
\hline
\end{tabular}

Evidence is accumulating in many areas of health care that higher case volume at a given institution usually confers improved outcomes. ${ }^{10}$ This has been specifically examined in pediatric cardiac surgery. ${ }^{11-13}$ Furthermore, the complexity of the Norwood operation has led to the suggestion that surgeons develop a learning curve. At the institutional level, programs have been developed to standardize the preoperative and postoperative care of these patients. The relative importance of each of these gained experiences

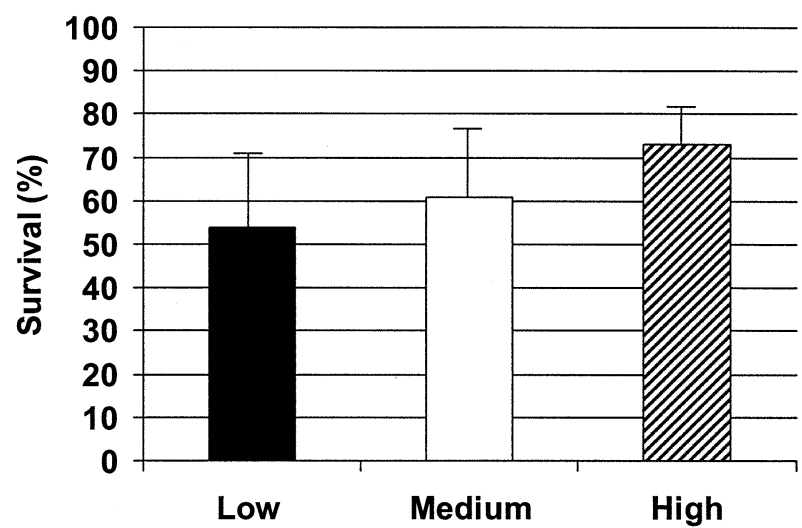

Figure 1. Survival ( \pm SD) versus institutional volume. Filled bar, Low-volume institutions; open bar, medium-volume institutions; hatched bar, higher-volume institutions $(P=.08)$.

remains undefined. The purpose of our study is to more clearly define the relative effect of institutional versus surgeon experience on the procedure outcome. Our hypothesis is that both institutional volume and surgeon experience play an important role in improved outcome after the Norwood procedure.

\section{Methods}

\section{Data Source}

This study used data from a large, multi-institutional administrative database, the Pediatric Health Information System (PHIS). The PHIS database is maintained by the pediatric hospital members of the Child Health Corporation of America (CHCA). CHCA is a business alliance of America's leading children's hospitals that provides a range of products and services designed to reduce cost, increase revenue, and enhance the competitive position of children's hospitals. This database contains member-specific standardized data on demographics, diagnoses, procedures, interventions, and outcomes for all pediatric patients admitted to each participating pediatric institution. For the analysis, we used the data from January 1, 1998, to December 31, 2001.

\section{Case Selection}

By using this database, the base population was defined as all patients with a principal diagnosis of HLHS (International Classification of Disease-ninth revision [ICD-9] diagnosis code 746.7) and age on admission of 30 days or less who were discharged from 1998 through 2001. The second stratification was determined by the existence of a Norwood procedure. There is not a specific ICD-9 or Current Procedural Terminology procedure code that indicates a Norwood procedure, and therefore the systemic-topulmonary arterial shunt (ICD-9 procedure code 39.0) in a patient with the principal diagnosis of HLHS was used as an indication that a Norwood procedure was performed. Other data fields included in the analysis were admission, discharge, and procedure dates; length of stay; final disposition; mortality; non-Norwood medical-surgical procedures; unique surgeons per institution; and medication use. 


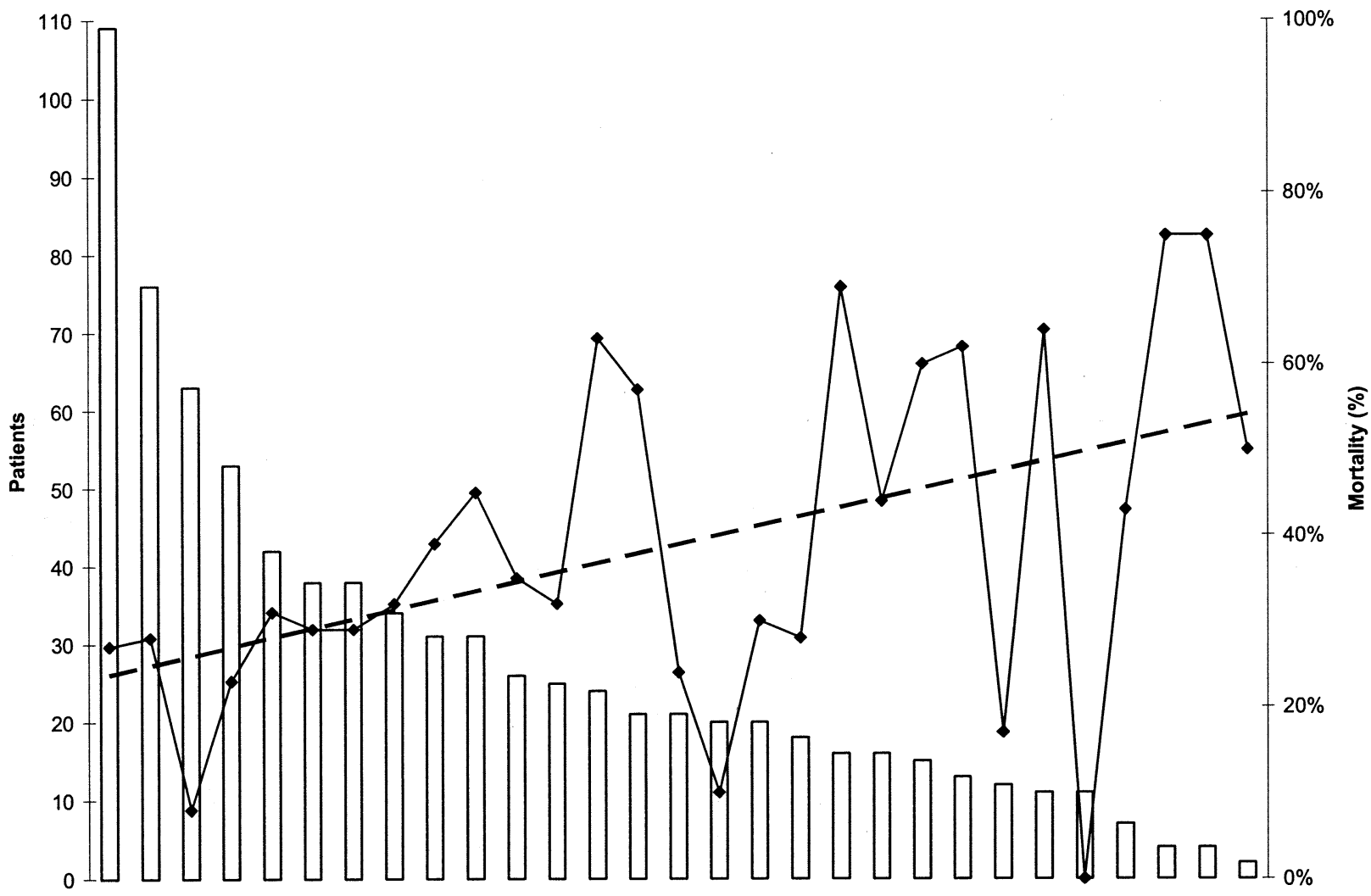

A

Institutions

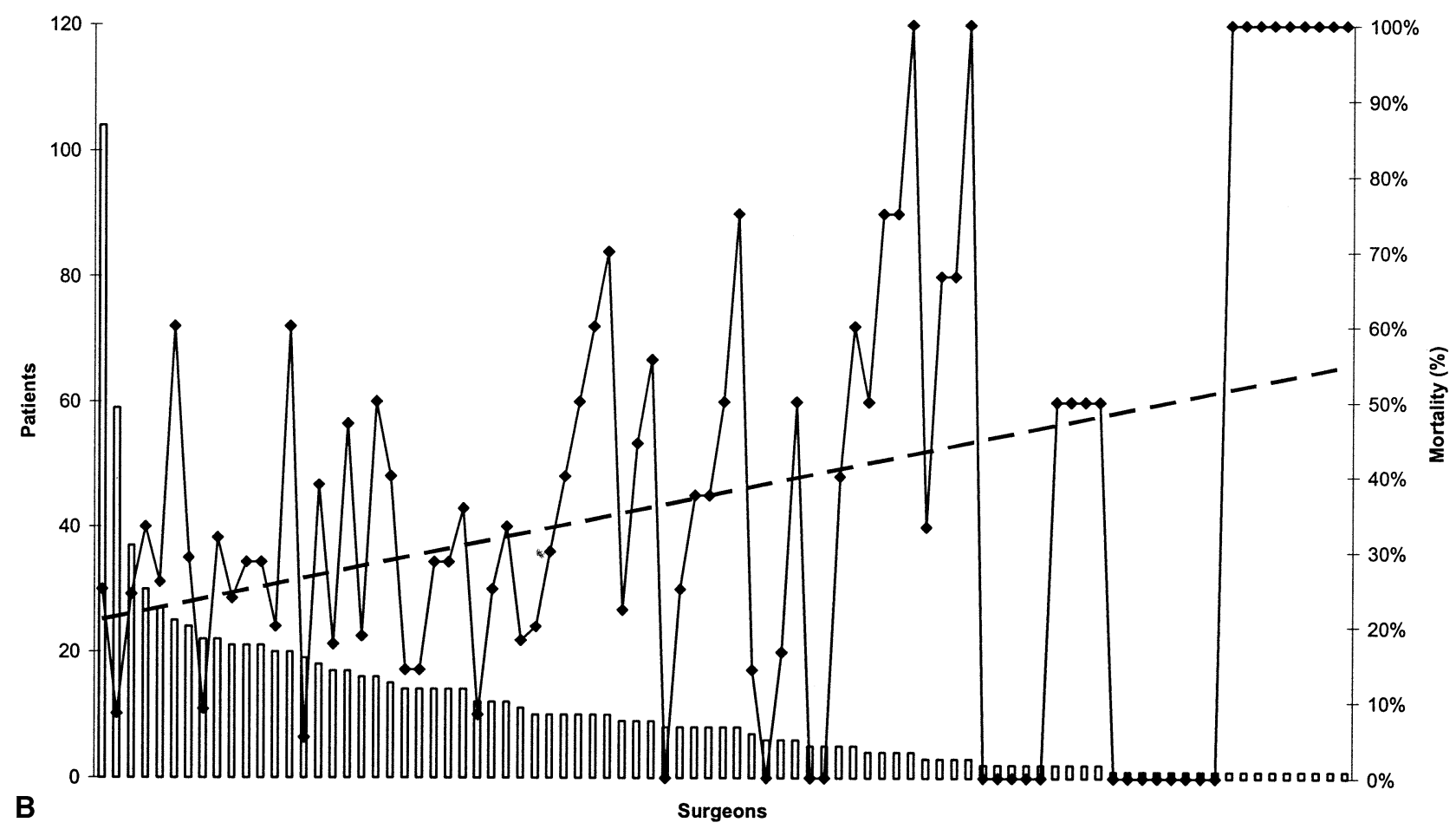

Figure 2. A, Mortality versus institutional volume. Open bars, Individual institutional volumes; solid line, individual institutional risk-unadjusted mortality; dashed line, mortality trend $(P=.02)$. B, Mortality versus individual surgeon volume. Open bars, Individual surgeon volumes; solid line, individual surgeon risk-unadjusted mortality; dashed line, mortality trend $(P=.13)$. 


\section{Data Analysis}

We conducted descriptive statistics to summarize the data. Stepwise multiple regression analysis was used to determine whether there was an association between the predictor variables: the institutional case volume, the number of cases performed by each surgeon, and the rate of risk-unadjusted mortality. All analyses were conducted with the use of the statistical software SAS version 10 (SAS Institute, Inc, Cary, NC).

\section{Results}

From January 1, 1998, to December 31, 2001, a total of 1105 children were given a diagnosis of HLHS, 801 of whom underwent surgical palliation with the Norwood procedure, 39 of whom underwent transplantation, and 265 of whom did not undergo an intervention. Twenty-nine hospitals within CHCA's pediatric institutions performed at least 1 Norwood procedure (range, 2-109 per institution). The total number of Norwood procedures performed at each institution is listed in Table 1 . The overall rate for survival to hospital discharge of infants undergoing the Norwood procedure was $68 \%(546 / 801)$.

To account for yearly fluctuations in patient volume, we categorized institutions on the basis of their total 4-year Norwood procedure volume: low, fewer than 16 total procedures; medium, 16 to 30 total procedures; and higher, greater than 30 total procedures. There were 9 hospitals in the low-volume category and 10 in the medium- and highervolume categories (Figure 1).

Survival ranged from $25 \%$ to $100 \%$ within the group of institutions. When examined as a categorical variable, the differences between low-, medium-, and higher-volume institutions were not significantly different. However, when examined as a continuous variable, survival after the Norwood procedure increased as the number of procedures performed by the institution increased (Figure 2). In the 4 of 29 institutions that averaged 1 or more Norwood procedure per month during the study period, survival averaged $78 \%$. The remaining 25 institutions averaged 1 Norwood procedure every 9.6 weeks, with a survival of $59 \%$.

A total of 87 surgeons performed at least 1 Norwood procedure during the 4 -year study period. The number of surgeons performing the Norwood procedure ranged from 1 to 6 per institution (Table 1). The average number of Norwood procedures performed per surgeon was 10.7 \pm 14 over the 4 -year period. This equated to 1 Norwood procedure every 19.4 weeks on average. There were 30 surgeons who performed 4 or fewer Norwood procedures during the study period, averaging to 1 or fewer procedures per year. The average survival after procedures performed by this group of surgeons was $49 \%$ (median, $50 \%$ ). This survival was not significantly different from that after procedures performed by higher-volume surgeons ( $>4$ Norwood procedures during the study period): $69 \% \pm 19 \%$ (median, 29\%). Only 2 of 87 surgeons
TABLE 2. Average length of stay of survivors, average time to death in nonsurvivors, and mean time to death in nonsurvivors by institutional category

\begin{tabular}{lccc}
\hline Institution & $\begin{array}{c}\text { Average length of } \\
\text { stay of survivors } \\
( \pm \mathbf{S D})\end{array}$ & $\begin{array}{c}\text { Average time } \\
\text { to death in } \\
\text { nonsurvivors } \\
( \pm \mathbf{S D})\end{array}$ & $\begin{array}{c}\text { Median time to } \\
\text { death in } \\
\text { nonsurvivors } \\
\text { (range) }\end{array}$ \\
\hline Low volume & $36.5 \pm 32.4$ & $19.6 \pm 33.2$ & $19.2(1-104)$ \\
Medium volume & $28.7 \pm 8.4$ & $12.2 \pm 9.7$ & $5.4(1-13)$ \\
Higher volume & $29.4 \pm 5.7$ & $20.2 \pm 9.6$ & $7.8(1-27)$
\end{tabular}

All values, $P=$ not significant.

averaged 1 or more Norwood procedures per month. Procedures by these 2 surgeons resulted in survivals of $75 \%$ and $92 \%$, respectively.

Results from the stepwise linear regression analysis revealed that lower risk-unadjusted mortality after the Norwood procedure was associated with higher institutional volume $\left(r^{2}=0.18, P=.02\right)$ but not between the number of procedures done by a surgeon and mortality $(P=.312)$. However, neither the institutional nor surgeon volume was associated with average length of stay in survivors or the time to mortality in nonsurvivors (Table 2). Survival after the Norwood procedure increased $4 \%$ (95\% confidence interval, $1 \%-7 \%$ ) for every 10 additional procedures performed over the 4-year study period per institution.

\section{Discussion}

It has been reported that increased experience with the complexities of the Norwood procedure yields improved outcomes. ${ }^{8}$ The importance of institution versus surgeon experience has not been examined. The purpose of this study was to examine the relationship between the mortality rate for surgical palliation of HLHS and the hospital and surgeon volumes from 1998 through 2001. Our study demonstrates a greater association between risk-unadjusted survival and the institutional surgical volume of Norwood procedures rather than the volume performed by individual surgeons.

Our findings agree with the results of previous studies examining the volume-outcome relationship in adult cardiac surgery. This has also been demonstrated in pediatric cardiac surgery. ${ }^{11,14}$ These reports, however, examined a broad range of congenital lesions and surgical procedures. A recent Institute of Medicine workshop ${ }^{15}$ reviewed 88 articles evaluating the relationship for 8 conditions and found that $79 \%$ of the studies demonstrated a positive effect of higher volume on outcome. Additional reports have confirmed that mortality was significantly reduced for 14 types of cardiovascular procedures at institutions performing a higher number of each procedure. ${ }^{10}$ 
The effect of institutional programs aimed at improvement in outcome has been previously demonstrated. Recently, we reported a significant improvement in survival after the Norwood procedure after the implementation of a preoperative and postoperative care plan involving an institutional commitment to change. ${ }^{9}$ The effect of institutional approaches to care has been demonstrated by others as well. Tweddell and colleagues ${ }^{2}$ reported a $93 \%$ survival after the Norwood procedure with the development and maintenance of a postoperative care pathway.

Individual surgeon experience has also been evaluated and has suggested an association between individual surgeon case volumes and outcome. ${ }^{12,16}$ In an editorial, Mavroudis and Jacobs ${ }^{17}$ appropriately state that although a general trend of higher-volume surgeons having improved outcomes might exist, many lower-volume surgeons and centers have excellent results. Although the statistical separation between the volume effects is small (Figure 2), this furthers supports the conclusion that the presence of surgeon experience is necessary but insufficient to account for improved outcome after this particular procedure.

Another important conclusion to be drawn from our data is that the Norwood procedure is a rare procedure, even in the highest-volume centers. Only 2 surgeons and 4 institutions performed more than 1 Norwood procedure per month when averaged over the 4-year study period. This might, in fact, explain our lack of determining an effect of case volume per surgeon; that is, perhaps there was inadequate volume difference seen between surgeons in this analysis to enable detection of such an effect. This suggests that programs and processes that are not only implemented but also sustained through constant re-education are necessary for success. Other authors have examined the role of regionalization of pediatric cardiac surgery. ${ }^{13}$ Our data suggest that such a strategy for this individual, high-risk procedure might improve outcome.

Our study was limited in that it did not include all pediatric surgical programs. Additionally, we lacked specific data on institutional approaches to the care of these patients. The PHIS database assigns a severity score to each patient and did not analyze any individual patient-level variables; therefore we were unable to quantify the effect of severity of individual cases or the role of comorbid conditions. Additionally, we were unable to account for nonintervention, transfer to another institution, and preoperative mortality in this type of analysis. Finally, a surrogate marker (ICD-9 code) for the Norwood procedure was used as inclusion criteria. Although the PHIS database is a financial database and suffers the potential inaccuracies of such a database, it provides one of the most complete samples of pediatric populations.
These results could lead to the expansion of the data set to include specific information from all institutions that perform the Norwood procedure. Additionally, these data suggest that there would be utility in formulating a multicenter examination of the success of standardized care pathways. Finally, the effect of newer surgical modifications, such as the Sano modification (right ventricle to pulmonary artery conduit), ${ }^{18}$ remains an unanswered question.

In conclusion, we demonstrate an association between risk-unadjusted survival after the Norwood procedure and institutional experience but not with individual surgeon case volume. These results emphasize the importance of improvements in institutional-based approaches to the care of children after the Norwood procedure.

\section{References}

1. Pearl JM, Nelson DP, Schwartz SM, Manning PB. First-stage palliation for hypoplastic left heart syndrome in the twenty-first century. Ann Thorac Surg. 2002;73:331-40.

2. Tweddell JS, Hoffman GM, Mussatto KA, Fedderly RT, Berger S, Jaquiss RDB, et al. Improved survival of patients undergoing palliation of hypoplastic left heart syndrome: lessons learned from 115 consecutive patients. Circulation. 2002;106:I82-9.

3. Poirier NC, Drummond-Webb JJ, Hisamochi K, Imamura M, Harrison AM, Mee RB. Modified Norwood procedure with a high-flow cardiopulmonary bypass strategy results in low mortality without late arch obstruction. J Thorac Cardiovasc Surg. 2000;120:875-84.

4. Jonas RA, Hansen DD, Cook N, Wessel D. Anatomic subtype and survival after reconstructive operation for hypoplastic left heart syndrome. J Thorac Cardiovasc Surg. 1994;107:1121-8.

5. Iannettoni MD, Bove EL, Mosca RS, Lupinetti FM, Dorostkar PC, Ludomirsky A, et al. Improving results with first-stage palliation for hypoplastic left heart syndrome. J Thorac Cardiovasc Surg. 1994;107: 934-40.

6. Bove EL. Current status of staged reconstruction for hypoplastic left heart syndrome. Pediatr Cardiol. 1998;19:308-15.

7. Bando K, Turrentine MW, Sun K, Sharp TG, Caldwell RL, Darragh RK, et al. Surgical management of hypoplastic left heart syndrome. Ann Thorac Surg. 1996;62:70-7.

8. Azakie T, Merklinger SL, McCrindle BW, Van Arsdell GS, Lee KJ, Benson LN, et al. Evolving strategies and improving outcomes of the modified Norwood procedure: a 10-year single-institution experience. Ann Thorac Surg. 2001;72:1349-53.

9. Checchia PA, Larsen R, Sehra R, Daher N, Gundry SR, Razzouk AJ, et al. Effect of a selection and postoperative care protocol on survival of infants with hypoplastic left heart syndrome. Ann Thorac Surg. 2004;77:477-83.

10. Birkmeyer JD, Siewers AE, Finlayson EV, Stukel TA, Lucas FL, Batista I, et al. Hospital volume and surgical mortality in the United States. N Engl J Med. 2002;346:1128-37.

11. Hannan EL, Racz M, Kavey RE, Quaegebeur JM, Williams R. Pediatric cardiac surgery: the effect of hospital and surgeon volume on in-hospital mortality. Pediatrics. 1998;101:963-9.

12. Jenkins K, Newburger J, Lock J, Davis R, Coffman G, Iezzoni L. In-hospital mortality for surgical repair of congenital heart defects: preliminary observations of variation by hospital caseload. Pediatrics. 1995;95:323-30.

13. Chang RK, Klitzner TS. Can regionalization decrease the number of deaths for children who undergo cardiac surgery? A theoretical analysis. Pediatrics. 2002;109:173-81.

14. Allen SW, Gauvreau K, Bloom BT, Jenkins KJ. Evidence-based referral results in significantly reduced mortality after congenital heart surgery. Pediatrics. 2003;112:24-8. 
15. Halm EA, Lee $C$, Chassin MR. Is volume related to outcome in health care? A systematic review and methodologic critique of the literature[summary for patients in Ann Intern Med. 2002;137(6):I52; PMID: 12230383]. Ann Intern Med. 2002;137:511-20.

16. Stark J, Gallivan S, Lovegrove J, Hamilton JR, Monro JL, Pollock JC, et al. Mortality rates after surgery for congenital heart defects in children and surgeons' performance. Lancet. 2000;355:1004-7.
17. Mavroudis C, Jacobs JP. Congenital heart disease outcome analysis: methodology and rationale. J Thorac Cardiovasc Surg. 2002;123: 6-7.

18. Sano S, Ishino K, Kawada M, Arai S, Kasahara S, Asai T, et al. Right ventricle-pulmonary artery shunt in first-stage palliation of hypoplastic left heart syndrome. J Thorac Cardiovasc Surg. 2003; 126:504-10.

To assure fairness to authors submitting work for consideration in The Journal of Thoracic and Cardiovascular Surgery, a mechanism exists for managing conflicts of interest. The editor and each of the section editors complete a "Conflict of Interest" form that identifies any and all relationships with commercial and other academic entities. When the editor has a potential conflict because of a relationship with another entity or author, the editor appoints an alternate editor from among the section editors or editorial board members who assumes the entire responsibility for final decisions on the manuscript in question. The editor does not read the reviews that are submitted nor engage in discussing the manuscript prior to the final decision. When the conflict of interest involves a section editor, a "guest section editor" is appointed who fills the role normally played by the conflicted section editor. All members of the editorial board and reviewers are asked to indicate any conflict of interest when they agree to review a manuscript. 